\title{
Allergic conjunctivitis and conjunctival provocation tests in atopic dogs
}

\author{
Ana Mafalda Lourenço-Martins, ${ }^{*}$ Esmeralda Delgado, ${ }^{*}$ Isabel Neto, ${ }^{*}$ Maria Conceição Peleteiro, \\ Mário Morais-Almeida† and José Henrique Duarte Correia* \\ ${ }^{*}$ CIISA, Faculdade de Medicina Veterinária, Universidade Técnica de Lisboa, Portugal; and †Department of Immunoallergology, Hospital Cuf-Descobertas, \\ Lisboa, Portugal
}

Address communications to:

E. Delgado

Tel.: 00351213652893

Fax: 00351213652822

e-mail: esmeralda@fmv.utl.pt

\begin{abstract}
Introduction Canine atopic dermatitis (cAD) is a very common disease, but little is known about eye involvement. The conjunctival provocation test (CPT) is used in human to study the ocular response to allergenic stimuli and to evaluate anti-allergic therapy. To our knowledge it has not been used in dogs.

Objectives To evaluate the prevalence of ocular signs in a population of atopic dogs and relate these with clinical $\mathrm{cAD}$ scores; and the usefulness of CPT for dust mites in atopic dogs with itchy eyes.

Procedures Sixty cAD patients were evaluated for (i) ocular signs of allergic

conjunctivitis including conjunctival hyperemia, chemosis, epiphora, ocular discharge, pruritus and corneal involvement, graded 0 to 3 according to severity, and (2) cAD Extent and Severity Index (CADESI-03). Additionally, CPTs for Dermatophagoides farinae $(n=12)$ and Dermatophagoides pteronyssinus $(n=12)$ were performed in sensitized atopic dogs and 24 control dogs.

Results Periocular and ocular signs of allergy were present in 60\% (36/60) of cases. Conjunctival hyperemia (90\%) was the most common sign. Severity of ocular signs was significantly correlated with eye pruritus $\left(r_{\mathrm{s}}=0.690, P=<0.001\right)$ and skin lesions score for head region $\left(r_{\mathrm{s}}=0.261, P=0.04\right)$. A highly significant difference $(P<0.001$, Fisher test) was found in CPTs between the test and the control groups.

Conclusion Allergic conjunctivitis signs associated with cAD seem under valuated so these patients would benefit from an ophthalmologic evaluation. Furthermore, we found CPT to be a reliable, easy to perform and safe test for the etiologic diagnosis of allergic conjunctivitis in the dog.
\end{abstract}

Key Words: allergic conjunctivitis, canine atopic dermatitis, conjunctival provocation test, Dermatophagoides farinae, Dermatophagoides pteronyssinus, house dust mites

\section{INTRODUCTION}

'Imagine life on the most exposed environment on the planet - the cornea'. ${ }^{1}$ In fact, as McMenamin describes in his inspiring article, the eye is subject to every kind of external aggression. So, it has developed the ability to adapt to many environmental changes, and protect itself from potentially hostile agents such as bacteria and viruses. Although it does not have lymphatic vessels, still possesses a rich immune system, ready to interact with antigens. ${ }^{2}$

An allergic disease is characterized by an exaggerated response to innocuous antigens, developed by genetically predisposed individuals. Allergic manifestations tend to occur where the body is most exposed to agents from the external environment. In humans the eye is often the target for allergic reactions. ${ }^{3}$ Allergic conjunctivitis is the most common form of eye allergy in human. The term 'allergic conjunctivitis' refers to a collection of hypersensitivity disorders that affect the lid, conjunctiva and/or cornea. Allergic conjunctivitis ranges in severity from mild to extremely severe forms, which can interfere significantly with quality of life and vision, imposing a substantial burden of disease and medical cost on allergy patients. ${ }^{4}$ Nevertheless, little focus is put to the ocular symptoms on their own. According to some human ophthalmologists the prevalence of ocular allergy clearly is underappreciated and has been under 
diagnosed and undertreated. ${ }^{2,4,5}$ Articles titles 'Allergic conjunctivitis: the forgotten disease' ${ }^{2}$ or 'Prevalence of allergic conjunctivitis: a missed opportunity', ${ }^{6}$ published by some of the most preeminent researchers in human ophthalmology, seem to say it all.

Diagnostic guidelines for the diagnosis of ocular allergic diseases in human are still inexistent. Mantelli and collaborators just set a new simple diagnostic algorithm to facilitate identification of the various clinical types of ocular allergic diseases and allow a more uniform approach to these patients. ${ }^{7}$ In the end, although the presumptive diagnosis of ocular allergy is mainly clinical, the final corroboration requires allergic testing. ${ }^{8}$ This is usually done by performing skin tests or serology to demonstrate sensitivity to one or more allergens, combined with interviewing the patient. However, only the conjunctival provocation test (CPT) provides clear evidence of a etiopathogenic relation between the conjunctival pathology and exposure to the allergen. ${ }^{8}$ This test is also useful to evaluate the antiallergic therapy efficacy. ${ }^{9}$ Efforts are being made to lead to the standardization of the CPT procedure for diagnostic purpose in human. ${ }^{8}$

A red eye is a common presentation in all veterinary clinical practices, with conjunctivitis being its most common cause. Conjunctivitis can be infectious (bacterial and viral), toxic/irritative or allergic in origin. Other forms of conjunctivitis are rare and related to systemic conditions, such as autoimmune diseases. Presumptive diagnosis of allergic conjunctivitis is made by exclusion of other causes of conjunctivitis, history and positive results on allergy testing (serology or intradermal tests). To our knowledge, we do not have standardized criteria, available to general practitioners and that would allow them to correctly and easily diagnose allergic conjunctivitis in the dog.

Although allergic conjunctivitis can probably exist on its own, it can also be concurrent to canine atopic dermatitis (cAD). In fact, the diagnosis of simultaneous $\mathrm{cAD}$ and conjunctivitis is not new. ${ }^{10}$ Recently, cAD was redefined as a genetically predisposed inflammatory and pruritic allergic skin disease with characteristic clinical features associated with $\mathrm{IgE}$ antibodies most commonly directed against environmental allergens. ${ }^{11} \mathrm{cAD}$ is clinically and immunologically similar to human atopic dermatitis. ${ }^{12}$ The exact prevalence of cAD remains unknown, but it is estimated, as in human, to affect around $10 \%$ of the population, with some breeds being more predisposed. ${ }^{13}$ The definition of cAD suggests strong breed and/or familial predispositions. Some dogs with cAD show concomitant nondermatologic signs, although few studies mentioned their prevalence. Three studies, done in the early 1980s by veterinarian dermatologists, found the prevalence of AC associated with cAD to be around $30 \%{ }^{14}$ and $50 \% .{ }^{15,16}$

The importance given to the ocular signs at that time must have been significant, as the presence of a bilateral conjunctivitis was even included as a minor criteria for the diagnosis of cAD according to Willemse criteria, published some years later. ${ }^{17}$ That interest seems to have vanished with time.

Since then, little has been reported about allergic conjunctivitis in cAD. In 2001, the American College of Veterinary Dermatology Task Force on Canine Atopic Dermatitis published an enlightening review of all that was known about $\mathrm{cAD}$ at that time. In the chapter on clinical signs it is mentioned that 'noncutaneous signs associated with $\mathrm{cAD}$ (such as rhinitis, sneezing or conjunctivitis) are reported to be uncommon or variable in their occurrence.' ${ }^{18}$ It is also stated that nondermatologic signs of canine atopy maybe severely underreported.

Earlier this year, Favrot and collaborators published a study with the characterization of the clinical features and signs of a large population of dogs $(n=843)$ with cAD. ${ }^{19}$ They looked for the presence of spring/summer conjunctivitis associated with $\mathrm{cAD}$ and found a prevalence of $21 \%$. Unfortunately no other ocular signs, such as perennial conjunctivitis, were reported. Most dogs in Europe have perennial symptoms, usually due to dust mite (Dermatophagoides farinae and/or Dermatophagoides pteronyssinus) sensitization, the most common allergens. ${ }^{20}$ In this study, the criteria used for the diagnosis of the allergic conjunctivitis are not described.

It is possible that allergic conjunctivitis in $\mathrm{cAD}$ is also underreported as seems to be the case in human. Maybe nondermatologic signs are also sometimes missed or undervalued as most dogs with $\mathrm{cAD}$ are seen by general physicians or dermatologists and not by ophthalmologists. Also, that we know of, CPTs have not been used to evaluate the contribution of specific allergens to allergic conjunctivitis in dogs with spontaneous cAD.

In this prospective study we aimed to (i) evaluate the prevalence of ocular signs in a population of dogs with cAD and relate their severity with skin signs, and (ii) to perform CPT with the dust mites Dermatophagoides farinae and Dermatophagoides pteronyssinus on a group of patients with $\mathrm{cAD}$, ocular allergy signs and sensitization to these mites. This will enable us to establish a relationship between ocular manifestations and the presence of a clinical relevant sensitization against these mites.

\section{MATERIALS AND METHODS}

The study was conducted in the teaching Hospital of the Faculty of Veterinary Medicine, Technical University of Lisbon. All the owners gave informed written consent for the inclusion of their dogs in the study.

\section{Phase 1 - "planet eye"}

Patient selection The study is of a prospective nature, and after it began all patients with cAD that were seen in the dermatology services were enrolled, after owners consent, independent of previous complaints or visits to our Hospital. Cases were enrolled and evaluated over the year. For each 
dog, diagnosis of cAD was made according to standard criteria. ${ }^{21,22}$ In summary, combination of (i) compatible history; (ii) fulfillment of clinical criteria strongly associated with the disease; (iii) exclusion of other pruritic skin diseases, specifically: no response to a minimum 8 -week diet trial with either a home-cooked or commercial hydrolyzed protein diet and water only to eliminate the possibility of an adverse food reaction; no response to a veterinary approved flea control regimen for at least 8 weeks; sarcoptic mange excluded by trial therapy and/or negative serology. In addition, the occurrence of at least one positive sensitization by intradermal test (IDT) reaction, performed in the skin of the lateral thorax and interpreted according to accepted criteria that agreed well with the patient's history was required. No antiinflammatory medication (topical or systemic) was given for at least 3 weeks prior to examination.

Dermatology Department evaluation All patients underwent a complete dermatologic evaluation and $\mathrm{CAD}$ clinical lesions were scored using the CADESI-03. This is a validated evaluation of clinical lesions (erythema, excoriation, lichenification, and self-induced alopecia) at 62 anatomical sites, each measured from 0 (normal) to 5 (most severe). ${ }^{23}$ Proposed intervals by the International Task Force on Canine Atopic Dermatitis for CADESI-03 are: remission: 0-15; mild AD: 16-59; moderate AD: 60-119; and severe $\mathrm{AD}: \geq 120 .{ }^{24}$

Additionally, one investigator (A.M. Martins) assessed if the dog had or not allergic conjunctivitis according to personal experience.

Ophthalmology Department evaluation A blinded, complete ophthalmic exam was performed in all dogs that included:

(1) external observation of the eye, eyelids and the peribulbar structures with a focal light source.

(2) tear production test assessed using Schirmer I Tear Test (STT, Dina strips Schirmer-Plus; Luneau SAS, Chartres, France) strips. Reference values were between 15 and $25 \mathrm{~mm} / \mathrm{min}^{25}$

(3) palpebral, direct and consensual pupillary reflexes as well as menace response, dazzle and oculocephalic reflexes evaluated in a dimly-lit room.

(4) measurement of the intraocular pressure (IOP) by applanation tonometry (Tono-Pen XL; Medtronic Solan, Jacksonville, FL, USA) following instillation of a drop of topical anesthetic (0.4\% Oxibuprocaine, Anestocil $^{\circledR}$; Oftalder, Oeiras, Portugal). Reference values were between 15 and $25 \mathrm{mmHg} .{ }^{25}$ The measurements were repeated three times. The values were considered valid when the coefficient of variance was $5 \%$ and the mean value was calculated.

(5) slit-lamp biomicroscopy using Kowa SL 15 (Kowa, Tokyo, Japan) performed in a darkened room. Ocular signs of allergic conjunctivitis were evaluated, including conjunctival hyperemia, chemosis, epiphora, ocular discharge, pruritus and corneal involvement, and graded 0 to 3 according to severity.

(6) funduscopy performed in a darkened room by indirect ophthalmoscopy (Heine Ómega 500, Herrsching, Germany) after dilatation of pupils with tropicamide (Tropicamide 1\%, Tropicil Top®; Edol, Linda-aVelha, Portugal).

(7) conjuctival brush cytology and fluorescein staining (Fluorescein; Haag-Streit International, Köniz, Switzerland) were performed to exclude other causes of ocular signs.

During ophthalmic examination ocular signs of allergic conjunctivitis including conjunctival hyperemia, chemosis, epiphora, ocular discharge, and corneal involvement were graded 0 to 3 according to severity.

Additionally, we asked the owners to evaluate their dog's pruritus according to the following classification: (0) no ocular pruritus; (1) mild ocular pruritus; (2) moderate ocular pruritus; (3) severe ocular pruritus. We have explained the owners that ocular pruritus can be manifested as ocular squinting, blepharospasm and attempts to scratch or rub their eyes with the paws or against objects. Sometimes they could even develop periocular alopecia as a result of self-inflicted trauma.

The final clinical score for the severity of the ocular signs resulted from sum of the individual scores (conjunctival hyperemia, chemosis, epiphora, ocular discharge, and corneal involvement and ocular pruritus) varying from 0 to 18 $(6 \times 3)$.

\section{Phase 2 - conjunctival provocation tests}

Patient selection Test Group: From the group of patients enrolled in phase 1 (Planet eye), 24 dogs with cAD, of which 12 were sensitized for Dermatophagoides farinae (Df) and other 12 to Dermatophagoides pteronyssinus (Dp), were selected as the test group. None of the dogs with cAD was having a flare of its allergy.

Control Group: The control group comprised two subgroups: (i) with 12 dogs with cAD but no mite sensitization on the IDT, and (ii) with 12 healthy dogs. All dogs were considered normal and bilaterally similar on previous ophthalmologic examination.

Conjunctival provocation tests To our knowledge there are currently no guidelines on how to perform CPTs in dogs, so we followed those for humans and the manufacturer's instructions. ${ }^{8}$ Briefly, we used ALK-Abelló Dermatophagoides farinae (Df) and Dermatophagoides pteronyssinus (Dp) lyophilized extract (Aquagen SQ; ALK-Abelló, Bioportugal, Algés, Portugal) and Aquagen specific diluent, water based and without phenol, to produce a $10 \mathrm{BU} / \mathrm{mL}$. Then, three serial dilutions of 1:5 were created, using sterile isotonic saline solution $(\mathrm{NaCl} 0.9 \%$, B.Braun Medical, Barcarena, 
Portugal) to arrive to the final testing solutions at the concentrations of $2,0.4$, and $0.08 \mathrm{BU} / \mathrm{mL}$.

All dogs underwent CPT as follows: aquagen diluent was used as the negative control and a single drop $(50 \mu \mathrm{L})$ was placed in the left conjunctival sac. A drop of successively stronger testing solutions was placed in the right conjunctival sac at 15-min intervals, each step in the test sequence being contingent on a negative response in the immediately preceding step. Fifteen minutes after each drop was instilled, both eyes were inspected for conjunctival hyperemia, chemosis and epiphora and each reaction scored as absent $=0$, mild $=1$, moderate $=2$ or severe $=3$. Ocular pruritus was also evaluated, using a scale with an extra score, extremely severe $=4$. The CPT was considered positive if the score was equal or higher to 5 (maximum 13) and implied no further testing. At the end of the test, a drop of dexametasone acetate in a $0.1 \%$ concentration (Dexaval- ${ }^{\circledR}$; Tecnifar, Lisboa, Portugal) was instilled in the right eye, to minimize discomfort and avoid late-onset reactions. All dogs were monitored for adverse reactions in the following $2 \mathrm{~h}$. The investigator (E. Delgado) was blinded for dogs' sensitization. Dogs from the control group had the CPT done for $\mathrm{Df}$ and $\mathrm{Dp}$ with more than 2 weeks interval in between.

\section{Statistical analysis}

Phase 1 (Planet eye) Descriptive statistics were calculated for sample description and characterization. Mean \pm standard deviation values for CADESI-03 and ocular score for the 60 patients of phase 1 "planet eye" are presented. Spearman Rank correlation was used to assess the relationship between ocular final score results, ocular pruritus, and severity of head and allover body skin lesions of cAD (evaluated by CADESI-03). Two-tailed Spearman's rho test was considered significant when $P<0.05$.

Phase 2 (Conjunctival provocation tests) CADESI-03 and ocular score values for the CPT test group are shown as mean \pm standard deviation. We used Fisher's exact test (a nonparametric test of significance) to test differences between groups of dogs sensitized either for $\mathrm{Df}$ or for $\mathrm{Dp}$ and the control subgroups (i) healthy dogs and (ii) cAD dogs. The data to which this test was applied are counts displayed in Table 1. Since we did not have an interval dependent

Table 1. Conjunctival provocation test results

\begin{tabular}{llcc}
\hline & Subgroup & $\begin{array}{c}\text { Positive } \\
\text { CPT (Df) }\end{array}$ & $\begin{array}{c}\text { Positive } \\
\text { CPT (Dp) }\end{array}$ \\
\hline Test group & cAD sensitized to Dp & n.a & $10 / 12$ \\
& cAD sensitized to Df & $11 / 12$ & n.a. \\
Control group & Healthy dogs & $0 / 12$ & $0 / 12$ \\
& cAD & $1 / 12$ & $1 / 12$ \\
\hline
\end{tabular}

$\mathrm{cAD}$, canine atopic dermatitis; $\mathrm{CPT}$, conjunctival provocation test; Df, Dermatophagoides farinae; Dp, Dermatophagoides pteronyssinus; n.a., Not applicable. variable, the ANOVA methods (parametric or nonparametric) could not be used.

Sensitivity, specificity, predictive positive value and predictive negative value with the respective $95 \%$ confidence intervals were calculated for both diagnostic tests (CPT for Df and CPT for Dp).

SPSS Statistics Release 17.0 (IBM Corporation, Somers, NY, USA) for Windows was used for all data analysis procedures.

\section{RESULTS}

\section{Phase 1 - "planet eye"}

Sixty atopic $\operatorname{dogs}(n=60)$ were selected according to standard criteria. The patients were enrolled and evaluated over the year, thereby representing a typical scenario in clinical practice. The majority of atopic dogs in Europe have all-year long clinical signs due to house dust mites allergy. Interestingly, we found out that 14 dogs enrolled in the study, had been previously sent by their general practitioner to our hospital ophthalmology service due to ocular pruritus and no primary eye problem was found. After that, and due to the dermatologic complaints, they referred them again, but this time to the dermatology service. They were at this point evaluated and after all criteria were checked, enrolled in this study.

Gender distribution was as follows: $45 \%(27 / 60)$ were females and $55 \%(33 / 60)$ males. The mean age was 5 years (mean deviation $=2.5)$. Twenty-one different breeds were included in a total of 44 purebred animals vs. 16 mongrel dogs. The most represented breeds were the Labrador retriever, the Golden retriever, the Yorkshire terrier, the Cocker spaniel and the Pug. All of these breeds have been found to have predisposition for atopic dermatitis. ${ }^{26}$

Mean \pm standard deviation values for CADESI-03 and ocular score for the 60 patients of phase 1 "planet eye" were respectively $87.2 \pm 72.03$ and $5.3 \pm 2.94$.

The most common ocular clinical signs were conjunctival hyperemia $90 \%$ and pruritus $73 \%$, followed by chemosis $70 \%$. Ocular discharge $60 \%$, epiphora $57 \%$ and corneal involvement $10 \%$ were less frequent.

The distribution of ocular signs and their relative severity can be seen in Fig. 1 and examples of these patients in Figs 2,3.

No signs of severe corneal ulceration (sterile/infective lesions) were observed in the six patients diagnosed with corneal involvement. One only presented corneal dystrophy and five had corneal neovascularization, from which two also presented punctuate keratitis and stained positive with fluorescein.

In the allergic conjunctivitis acute cases there was epiphora, conjunctival hyperemia, squinting and a certain degree of blepharospasm while in the chronic states the animal evidenced follicular conjunctivitis with hypertrophy of lymphoid follicles visible in the bulbar conjunctiva and inner part of the third eyelid as well as the presence of a white mucus discharge. 


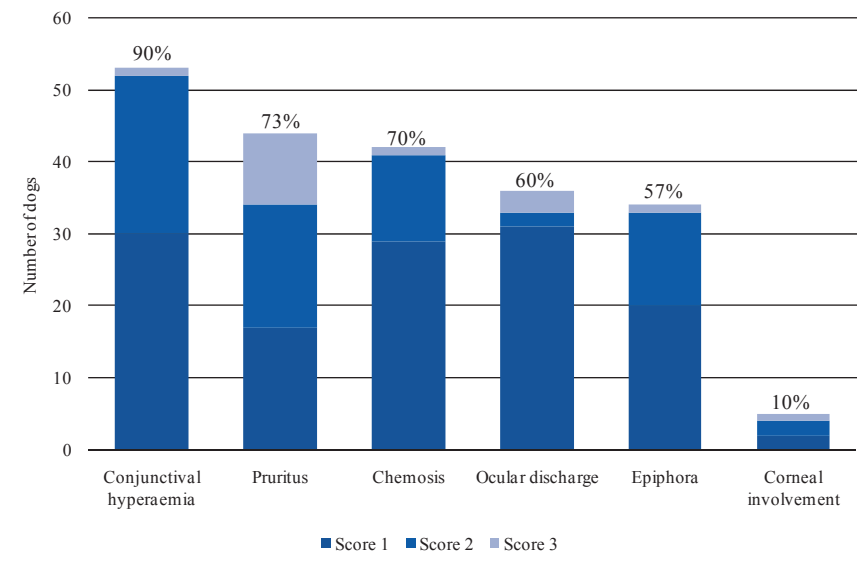

Figure 1. Ocular signs score in cDA patients $(n=60)$. The graphic shows the percentage and the number of animals graded as mild (score 1), moderate (score 2 ) or severe (score 3 ).

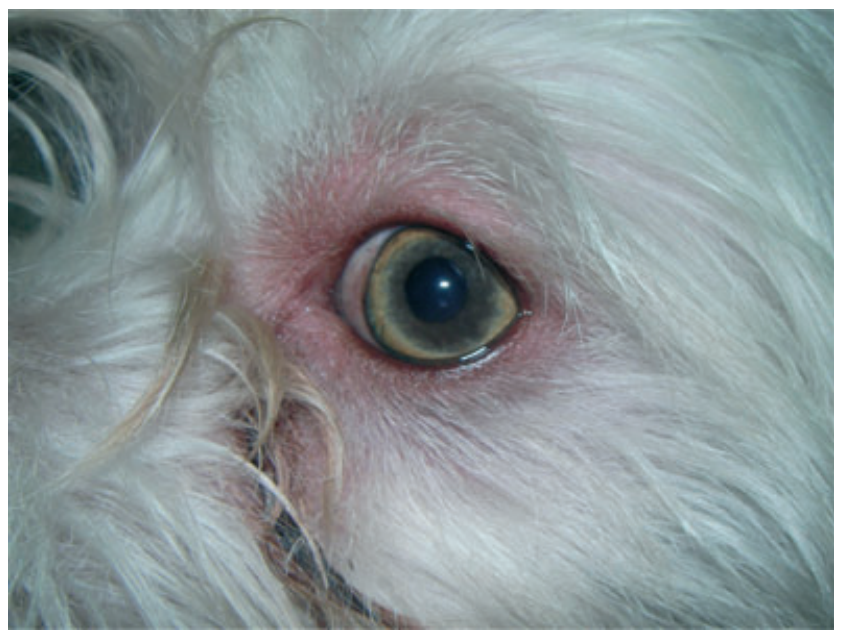

Figure 2. Signs of active allergic blepharitis and conjunctivitis: periocular alopecia and congestion, blepharitis and epiphora in the right eye of an atopic dog with ocular pruritus. Clinical ocular score of 8. CADESI-03 of 30, compatible with a mild canine atopic dermatitis (cAD).

The prevalence of allergic conjunctivitis varied with the department: ophthalmology diagnosed $36 / 60(60 \%)$ and dermatology only $10 / 60$ (17\%). So, dermatology missed to diagnose 26 cases $(72 \%)$ of allergic conjunctivitis cases according to ophthalmologic evaluation.

Evidence of a significant relationship between eye pruritus and final ocular score was found (Spearman correlation coefficient $\left.r_{\mathrm{s}}=0.690 ; P<0.001\right)$. Skin lesion score for the region of the head and final ocular score were also significantly correlated $\left(r_{\mathrm{s}}=0.261 ; P=0.04\right)$. However, data provided no evidence of a relationship between CADESI-03 and final ocular score $\left(r_{\mathrm{s}}=-0.024 ; P=0.86\right)$, which means that we could not find any association between severe form of dermatitis and conjunctivitis.

\section{Phase 2 - conjunctival provocation tests}

CADESI-03 and ocular score values for the CPT test group Df are respectively $89.9 \pm 85.46$ and $3.6 \pm 2.02$ (shown as mean \pm standard deviation); for Dp group are correspondingly $64.3 \pm 41.1$ and $4.17 \pm 2.62$. The results of the CPT can be observed in Table 1. The comparison of results between the test group sensitized to Df and the control group (both sub-group healthy dogs and sub-group cAD), yielded significant differences (in both cases $P<0.001$, twosided Fisher's exact test). Similarly, the test group sensitized to Dp yielded the same results when compared with both control sub-groups $(P<0.001$, two-sided Fisher's exact test).

The diagnostic sensitivity and specificity of CPT for dust mite Df were, respectively $91.7 \%$ (95\% CI 67.8 to $99.3 \%$ ) and $95.8 \%$ (95\% CI 83.9 to $99.6 \%$ ). The corresponding positive predictive value (PPV) was $91.7 \%$ (95\% CI 67.8 to $99.3 \%$ ) and the negative predicted value (NPV) was $95.8 \%$ (95\% CI 83.9 to $99.6 \%$ ). The same calculations performed for diagnostic CPT for Dp yielded: a sensitivity of $83.3 \%$ (95\% CI 58.9 to $91.2 \%$ ), a specificity of $95.8 \%$ (95\% CI 83.6 to $99.8 \%$ ), a PPV of $90.9 \%$ (95\% CI 64.2 to $99.5 \%$ ) and a NPV of $92 \%$ (95\% CI 80.3 to $95.8 \%$ ).

The majority of animals $(71 \%, 15 / 21)$ had positive CPT only with the most concentrated solution $(2 \mathrm{BU} / \mathrm{mL})$, four scored positive with the medium dilution $(0.4 \mathrm{BU} / \mathrm{mL})$ while only two scored positive with the mildest dilution (0.08 BU/mL).

Detailed information on the results obtained in the test group can be seen in Fig. 4. In Fig. 5, we can see an example of a positive CPT in a sensitized atopic dog.

\section{CPTs side effects}

Adverse side effects were noticed only in two animals from the test group sensitized to Df $(8 \% ; 2 / 24)$. These dogs showed a sudden exacerbation of their atopic state (a flare), and were observed again by clinicians from both ophthalmology and dermatology departments. Both dogs had marked increases on CADESI-03 and pruritus (ocular and global). In both cases, the dogs had initial CADESI-03 values compatible with severe disease (CADESI-03 of 126 and $153)$ and an ocular score of 5 and 7 and no corneal involvement in any of the cases). An oral anti-inflammatory therapy was prescribed (Prednisolone, $1 \mathrm{mg} / \mathrm{kg} / \mathrm{SID}, 5$ days) and 3 days after the episode they were both controlled and therapy was discontinued. No other dogs showed any side effect.

\section{DISCUSSION}

Canine atopic dermatitis is a common condition in dogs and its prevalence seems to be increasing over the last decades. Overall, the exact prevalence of allergic conjunctivitis in $\mathrm{cAD}$ is unknown. According to our results, $\mathrm{cAD}$ concomitant eye allergy is more frequent than mentioned in the literature, ${ }^{18}$ and its prevalence seem to be strongly influenced by the veterinarian's individual area of specialization.

This results resemble those observed by Bonini regarding medical class. ${ }^{5}$ Conjunctivitis prevalence among human allergic patients seems highly dependent on the doctors' 
Figure 3. Right and left eye of a severely affected atopic dog with periocular alopecia and congestion due to blepharitis with intense pruritus and conjunctival hyperemia, congestion and chemosis. Below we can see the result of the intense pruritus with licking dermatitis and self excoriations lesions in the posterior paws of the same patient and an example of a positive intradermal skin test. Clinical ocular score of 10. CADESI-03 of 350, compatible with severe canine atopic dermatitis (cAD).
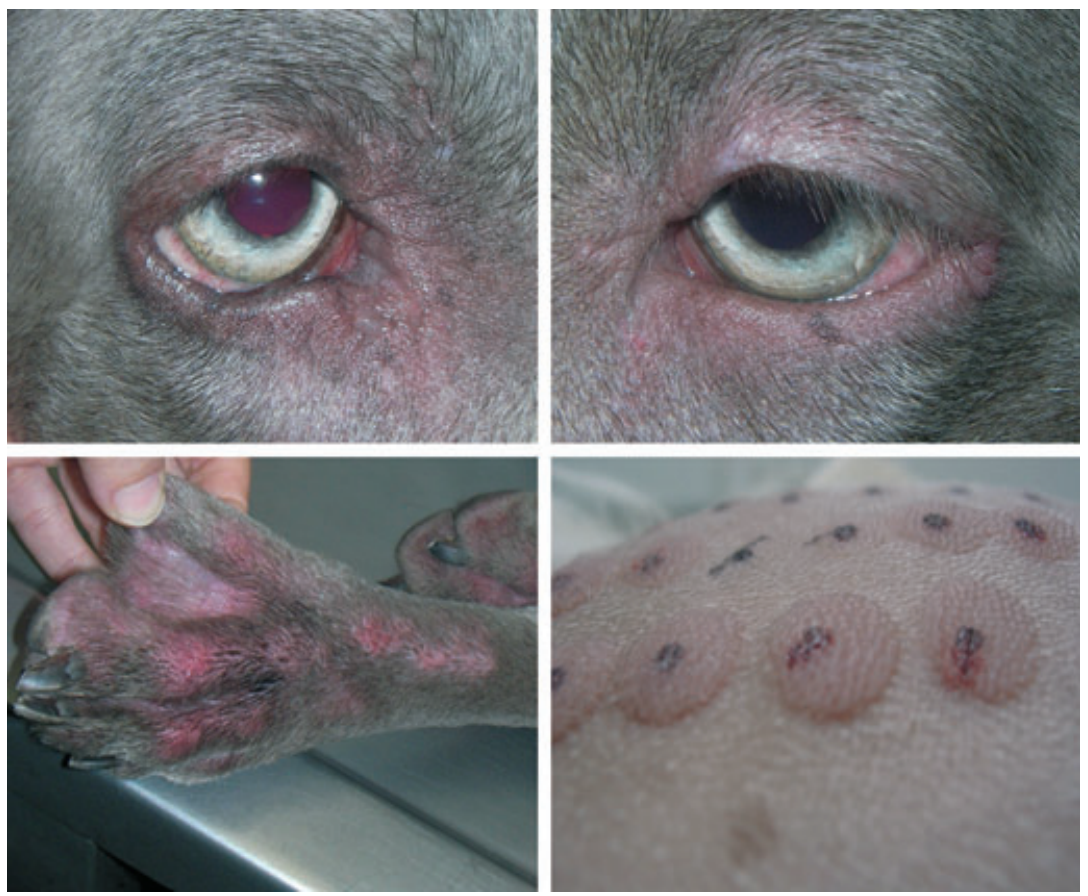

CPT results

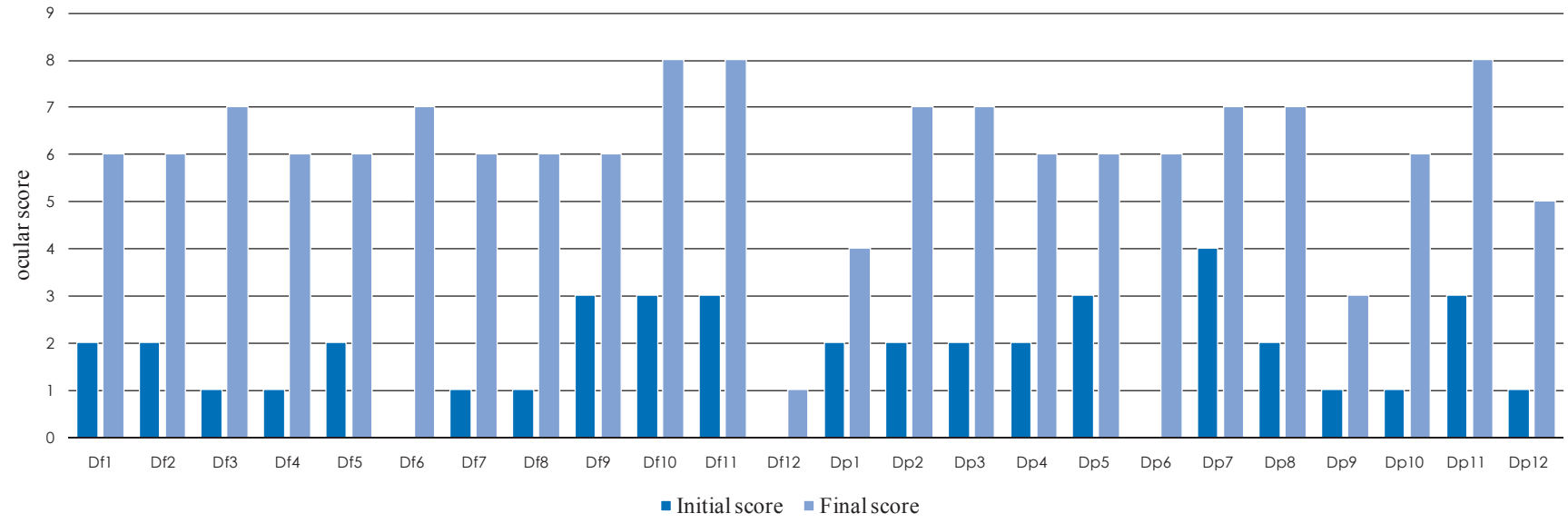

Figure 4. Initial and final score values obtained on conjunctival provocation tests (CPTs) for Dermatophagoides farinae (Df, $n=12)$ and Dermatophagoides pteronyssinus ( $\mathrm{Dp}, n=12$ ) in sensitized canine atopic dermatitis (cAD) patients.

speciality. In an unpublished study by Bonini and collaborators, 2206 allergic human patients were observed by allergists, dermatologists, ophthalmologists and pneumologists, according to a specialist approach. For the same patients, diagnosis of conjunctivitis was made in 45\% (allergists), $10 \%$ (dermatologists), 90\% (ophthalmologists) and 25\% (pneumologists) of the patients. Clearly, the variety of results reflects individual main professional focus.

It is possible that allergic conjunctivitis in $\mathrm{cAD}$ is also underreported. Maybe nondermatologic signs are sometimes missed or under valuated as most dogs with $\mathrm{cAD}$ are seen by general physicians or dermatologists and not by ophthalmologists. Also, attention can be distracted from the eye due to more severe skin signs. Additionally, ocular pruritus can be difficult to evaluate, both by owners and veterinarians, as dogs with cAD usually tend to rub or scratch the face also due to ear, muzzle and perilabial pruritus, making it difficult to point the finger. Lastly, the majority of $\mathrm{cAD}$ patients are medicated with systemic anti-inflammatory (corticosteroid and cyclosporine) therapeutics which will also control allergic ocular signs.

We believe that the existence of standardized criteria available to general veterinary physicians and dermatologists would allow them to correctly and easily diagnose allergic conjunctivitis, especially in those cases that do not need an ophthalmologic referral.

Our results show a correlation between the severity of ocular signs and both eye pruritus and skin lesions score for 


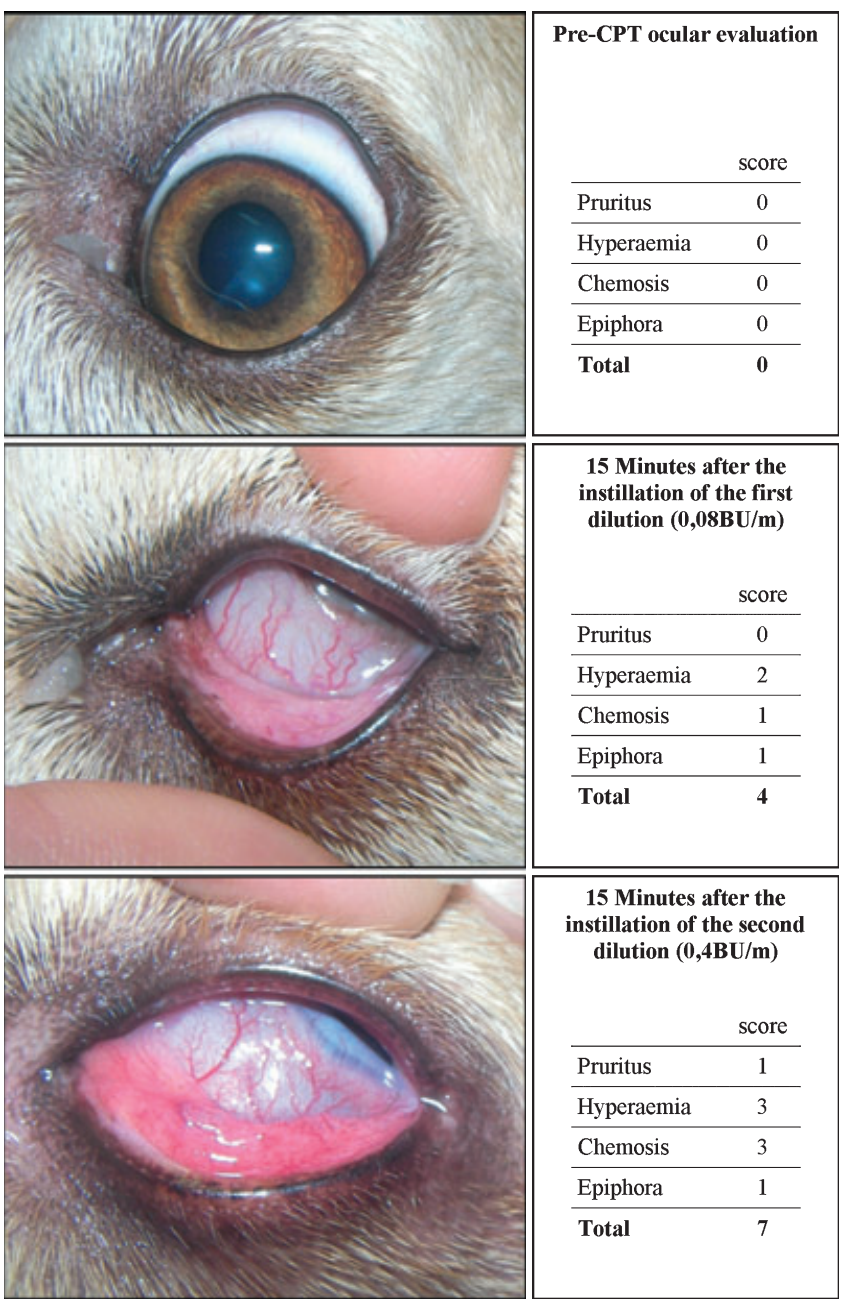

Figure 5. Positive conjunctival provocation test (CPT) in a sensitized atopic dog.

the head: ocular pruritus can indirectly result in more severe skin lesions for that area.

Overall severity of cAD global skin lesions did not correlate with the severity of ocular signs or pruritus, so we could not find any association between severe form of dermatitis and conjunctivitis. It is possible that certain areas of the body are 'pre-programmed' to develop inflammation upon allergen challenge once sensitization has occurred. ${ }^{27}$ This may be individually tailored to some extent, with some patients developing more important skin or eye signs. People sensitized for the same allergens will respond with dermatitis, rhinitis, conjunctivitis or an association of these. We believe this can also be the case in dogs.

We took 24 cAD sufferers with Dp or Df sensitization, we performed CPT for both dust mites and we compared the results obtained in the test group with a control group of healthy dogs and atopic dogs without dust mite sensitivity. A very significant relation $(P<0.001$; Fisher exact test) was found between the presence of dust mite sensitization and positive CPT, showing a causal relationship between these allergens and the ocular signs in these patients.
The diagnostic sensitivities and specificities for each CPT (Dp and Df) were high, and similar to what was found by others for humans. ${ }^{28}$

Results from a recent study done with high IgE-producing Beagle dogs (an experimental model for atopic dermatitis, sensitized both to Dp and Df, corroborate our findings that these allergens can, in sensitized individuals, originate allergic conjunctivitis upon exposure. These dogs were submitted to environmental, specific oral and epicutaneous provocation tests with Dp and Df. Regardless of the route used, all developed periocular eritema. Two of six dogs, $33 \%$ of the population studied, developed allergic conjunctivitis after the environmental exposure.

We found CPT to be rapid and easy to perform, without the need of sedation. Also, we think it can be considered an innocuous provocation test, given some precaution measures are taken. In our case, all tests were performed in a hospital environment and the animals were closely monitored by two investigators (A.M. Martins and E. Delgado) for any adverse reactions until $2 \mathrm{~h}$ after the test. Furthermore, a drop of a potent topical corticosteroid was applied after the test was completed to the allergen-tested eye.

Although every effort was taken to prevent side effects, two dogs had a sudden exacerbation of their allergic diseases. It is not possible to definitely conclude that the CPT was the cause of their acute clinical deterioration, but knowing that these dogs had been stable for some time, and no other conditions changed, we suspect that some systemic absorption of the allergens might have occurred and triggered the allergic response. Even if uncommon, flares of atopic states have been described in human after $\mathrm{CPT}^{8}{ }^{8}$ Besides the measures already taken, we think it may be wise to provide the owner with an oral form of corticosteroid to use in case of a flare after the CPT, especially in patients with moderate to severe forms of $\mathrm{cAD}$.

We think absorption by the conjunctiva, as well as exposure of the respiratory mucosa from nasal cavity to allergens due to transport through the nasolacrymal duct, could result in an increased allergen exposure. Several studies in people point out that topical treatment for rhinitis allergy also ameliorates conjunctivitis symptoms and vice versa, advocating for the importance of this communication route. ${ }^{4,29,30}$

Furthermore with CPT we were able to establish an etiologic relationship between ocular manifestations and specific mite sensitizations. With CPT, a link could be made between conjunctival pathology and exposure to allergens for which the patient is known to be sensitized. These tests are considered specific, and are probably the simplest way to confirm or rule out specific allergic components of conjunctivitis. Further studies, namely with other allergens, are warranted to evaluate CPT potential as a gold-standard test for the etiologic diagnosis of allergic conjunctivitis in the dog but CPT seems to be a promising tool for the etiologic diagnosis of allergic conjunctivitis in $\mathrm{cAD}$ patients, especially for those with significant eye symptoms. The identification of the cause of allergic conjunctivitis by CPT 
will allow a better understanding of this disease, and opens new therapeutic monitorization tools. This is especially important for those patients that have essentially ocular symptoms and are in need of chronic anti-inflammatory treatment with topical or systemic forms of corticosteroids or calcineurin inhibitors. It would be particularly interesting to see if these patients could benefit from specific immunotherapy.

The European Medicines Agency (EMA) states that for human the CPT is a validated model for studying allergic conjunctivitis. ${ }^{31}$ The provoked reaction can be used to evaluate any allergic topical ocular products, with the benefit of the patient's contralateral eye acting as his/her own control. ${ }^{31}$ Furthermore, the CPT evaluating the response to an allergen challenge before and after the effect of an antiallergic agent as compared with placebo may be used as supportive evidence for efficacy and to establish a dose effect relationship. ${ }^{31}$ In fact, most of the knowledge on the efficacy of certain drugs for eye allergy in human was obtained through CPT. ${ }^{32}$ In our opinion, CPT should be evaluated as a tool for the evaluation of the efficacy of topical ocular medications for eye allergy in the dog as well.

Nevertheless further studies with larger populations and other allergens are needed to completely establish the usefulness of CPT in dogs with ocular allergy.

We strongly believe that cooperation between ophthalmologists and dermatologists would be of benefit for these patients, resulting in a multifaceted comprehension of $\mathrm{cAD}$, especially the role of ocular signs as a co-morbility of cAD and a better evaluation and control of their clinical signs, including the use of topical ocular forms of therapy to help control their itchy eyes, enhancing their quality of life.

Little is known about eye allergy in the dog. It seems to have features that resemble their human counterparts, lifting the veil for a possible suitable model for the study of this disease in human. But first, a better characterization of this disease in the dog is needed, and would probably benefit from uniform diagnostic criteria and use of CPTs, maybe more properly designated by conjunctival allergen tests.

\section{ACKNOWLEDGMENTS}

The authors want to thank all the owners who accepted to be a part of this project, and final year students that helped enthusiastically with the clinical work. We are also grateful to CIISA (Centro de Investigação Interdisciplinar em Sanidade Animal) and Portuguese Foundation of Science and Technology (FCT). We also thank Bioportugal, ALK-Abelló for allergen extracts.

\section{REFERENCES}

1. McMenamin PG. Ida Mann Lecture 2007: Planet eye. Clinical and Experimental Ophthalmology 2008; 36: 592-599.

2. Bonini S. Allergic conjunctivitis: the forgotten disease. Chemical Immunology and Allergy 2006; 91: 110-120.
3. Leonardi A, Motterle L, Bortolotti M. Allergy and the eye. Clinical and Experimental Immunology 2008; 153(Suppl. 1): $17-21$.

4. Bielory L. Allergic conjunctivitis and the impact of allergic rhinitis. Current Allergy and Asthma Reports 2010; 10: 122-134.

5. Bonini S. Allergic conjuctivitis. 1st International Congress of Southern European Allergy Societies. Florence, Italy, 2010.

6. Riedi CA, Rosario NA. Prevalence of allergic conjunctivitis: a missed opportunity? Allergy 2010; 65: 131-132.

7. Mantelli F, Lambiase A, Bonini S. A simple and rapid diagnostic algorithm for the detection of ocular allergic diseases. Current Opinion in Allergy and Clinical Immunology 2009; 9: 471-476.

8. Mortemousque B, Fauquert JL, Chiambaretta F et al. Conjunctival provocation test: recommendations. Fournal Francais $d$ Ophtalmologie 2006; 29: 837-846.

9. Mortemousque B. Conjunctival provocation test. Fournal Francais d Ophtalmologie 2007; 30: 300-305.

10. Wittich FW. Spontaneous allergy (atopy) in the lower animal. Journal of Allergy 1941; 12: 247-251.

11. Halliwell RE. Revised nomenclature for veterinary allergy. Veterinary Immunology and Immunopathology 2006; 114: 207-208.

12. Marsella R, Girolomoni G. Canine models of atopic dermatitis: a useful tool with untapped potential. Fournal of Investigative Dermatology 2009; 129: 2351-2357.

13. Hillier A, Griffin CE. The ACVD task force on canine atopic dermatitis (I): incidence and prevalence. Veterinary Immunology and Immunopathology 2001; 81: 147-151.

14. Willemse A, van den Brom WE. Investigations of the symptomatology and the significance of immediate skin test reactivity in canine atopic dermatitis. Research in Veterinary Science 1983; 34: 261-265.

15. Scott DW. Observations on canine atopy. Fournal of the American Animal Hospital Association 1981; 17: 91-100.

16. Willemse A. Canine atopic disease: investigations of eosinophils and the nasal mucosa. American fournal of Veterinary Research 1984; 45: 1867-1869.

17. Willemse T. Atopic dermatitis in dogs: current diagnostic criteria. Tijdschrift Voor Diergeneeskunde 1988; 113: 74-79.

18. Griffin CE, DeBoer DJ. The ACVD task force on canine atopic dermatitis (XIV): clinical manifestations of canine atopic dermatitis. Veterinary Immunology and Immunopathology 2001; 81: 255269.

19. Favrot C, Steffan J, Seewald W et al. A prospective study on the clinical features of chronic canine atopic dermatitis and its diagnosis. Veterinary Dermatology 2010; 21: 23-31.

20. Hill PB, DeBoer DJ. The ACVD task force on canine atopic dermatitis (IV): environmental allergens. Veterinary Immunology and Immunopatbology 2001; 81: 169-186.

21. Hillier A, DeBoer DJ. The ACVD task force on canine atopic dermatitis (XVII): intradermal testing. Veterinary Immunology and Immunopathology 2001; 81: 289-304.

22. DeBoer DJ, Hillier A. The ACVD task force on canine atopic dermatitis (XV): fundamental concepts in clinical diagnosis. Veterinary Immunology and Immunopathology 2001; 81: 271-276.

23. Olivry T, Marsella R, Iwasaki T et al. Validation of CADESI-03, a severity scale for clinical trials enrolling dogs with atopic dermatitis. Veterinary Dermatology 2007; 18: 78-86.

24. Olivry T, Mueller R, Nuttall T et al. Determination of CADESI03 thresholds for increasing severity levels of canine atopic dermatitis. Veterinary Dermatology 2008; 19: 115-119.

25. Gelatt K. Ophthalmic examination and diagnostic procedures. Essentials of Veterinary Ophthalmology. Lippincott Williams and Wilkins, Philadelphia, 2000; 1-26. 
26. Sousa CA, Marsella R. The ACVD task force on canine atopic dermatitis (II): genetic factors. Veterinary Immunology and Immunopathology 2001; 81: 153-157.

27. Marsella R, Nicklin C, Lopez J. Studies on the role of routes of allergen exposure in high IgE-producing beagle dogs sensitized to house dust mites. Veterinary Dermatology 2006; 17: 306-312.

28. Bertel F, Mortemousque B, Sicard H et al. Conjunctival provocation test with Dermatophagoides pteronyssinus in the diagnosis of allergic conjunctivitis from house mites. Fournal français d'ophtalmologie 2001; 24: 581-589.

29. Prenner BM, Lanier BQ, Bernstein DI et al. Mometasone furoate nasal spray reduces the ocular symptoms of seasonal allergic rhinitis. Fournal of Allergy and Clinical Immunology 2010; 125: 1247-1253, e5.
30. Crampton HJ. A comparison of the relative clinical efficacy of a single dose of ketotifen fumarate $0.025 \%$ ophthalmic solution versus placebo in inhibiting the signs and symptoms of allergic rhinoconjunctivitis as induced by the conjunctival allergen challenge model. Clinical Therapeutics 2002; 24: 1800-1808.

31. The European Agency for the Evaluation of Medicinal Products (EMEA). CHMP/EWP/2455/02. Guideline on the clinical development of medicinal products for the treatment of allergic rhinoconjunctivitis. EMEA, London, UK, 2002.

32. Friedlaender $\mathrm{MH}$. Conjunctival provocation testing: overview of recent clinical trials in ocular allergy. Current Opinion in Allergy and Clinical Immunology 2002; 2: 413-417. 